ARTICLE

Received 29 Dec 2014 | Accepted 16 Jun 2015 | Published 28 Jul 2015

DOI: $10.1038 /$ ncomms 8831

OPEN

\title{
Infrared nanospectroscopy characterization of oligomeric and fibrillar aggregates during amyloid formation
}

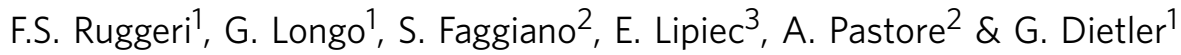

Amyloids are insoluble protein fibrillar aggregates. The importance of characterizing their aggregation has steadily increased because of their link to human diseases and material science applications. In particular, misfolding and aggregation of the Josephin domain of ataxin-3 is implicated in spinocerebellar ataxia-3. Infrared nanospectroscopy, simultaneously exploiting atomic force microscopy and infrared spectroscopy, can characterize at the nanoscale the conformational rearrangements of proteins during their aggregation. Here we demonstrate that we can individually characterize the oligomeric and fibrillar species formed along the amyloid aggregation. We describe their secondary structure, monitoring at the nanoscale an $\alpha$-to- $\beta$ transition, and couple these studies with an independent measurement of the evolution of their intrinsic stiffness. These results suggest that the aggregation of Josephin proceeds from the monomer state to the formation of spheroidal intermediates with a native structure. Only successively, these intermediates evolve into misfolded aggregates and into the final fibrils.

\footnotetext{
${ }^{1}$ Laboratoire de Physique de la Matière Vivante, Ecole Polytechnique Fédérale de Lausanne (EPFL), CH-1015 Lausanne, Switzerland. ${ }^{2}$ Department of Basic and Clinical Neuroscience, King's College, London, UK. ${ }^{3}$ Institute of Nuclear Physics, Polish Academy of Sciences, 31-342 Krakow, Poland. Correspondence and requests for materials should be addressed to A.P. (email: annalisa.pastore@crick.ac.uk) or to G.D. (email: giovanni.dietler@epfl.ch).
} 
A ging of the population has increased the visibility of several neurodegenerative disorders such as Parkinson's, Huntington's and Alzheimer's diseases ${ }^{1}$. Their onset is connected with insoluble fibrillar protein aggregates, called amyloids $^{2}$. However, these structures are not only associated with diseases since they were also discovered in many physiologically beneficial roles (functional amyloids) including bacterial coatings, adhesives and structures for the storage of peptide hormones ${ }^{3-5}$. During their aggregation, monomeric proteins undergo internal structural rearrangements leading to the formation of fibrils with a universal cross $\beta$-sheet quaternary structure ${ }^{6}$. This conformation is independent of the monomeric initial structure and is the fingerprint of amyloids.

The aggregation pathway typically proceeds through the formation of oligomers and protofibrils, which lead to the mature fibrils ${ }^{7}$. Strong evidence indicates that neurodegeneration and cytotoxicity are produced by the intermediate species of fibrillization rather than by the end point. This poses the problem of investigating the early stages of the interconversion of monomers into oligomers and amyloid fibrils ${ }^{8}$. An important question is whether misfolding into $\beta$-rich structures precedes or follows aggregation. This is not a trivial point, since the conversion of normally folded proteins into fibrils is generally assumed to be caused by partially misfolded conformations exposing amyloidogenic regions, otherwise protected in the hydrophobic core. A fascinating hypothesis is that not all proteins need to undergo misfolding before aggregation can start. There might be cases in which aggregation proceeds through formation of native aggregates, which undergo misfolding only in an advanced stage. One of the proteins suggested to have this behaviour is the Josephin domain of ataxin-3 (ref. 9). This human protein is of wide interest because its aggregation is responsible for spinocerebellar ataxia of type 3 (refs 10-13). This hypothesis remains open since, with the currently available techniques, it was impossible to investigate structurally the aggregate intermediate and final species. Its testing requires the use of new characterization methodologies because of the complexity of the energy landscape that leads to aggregation.

Infrared nanospectroscopy (nanoIR) $)^{14,15}$ is an innovative tool that exploits the combination of two techniques commonly used to study protein aggregation: atomic force microscopy (AFM) and infrared spectroscopy. The first can provide information on the morphology and mechanical properties of the species formed along the aggregation pathway ${ }^{16-18}$, the second can characterize conformational changes in protein secondary structure ${ }^{19-22}$. Although useful, these conventional techniques do not tell us separately whether/at which time point misfolding occurs, nor what is the correlation between nanomechanical properties and secondary structure of the individual species. Their combination, in nanoIR, enables a structural characterization of the properties of amyloids and the discrimination of objects in the same AFM map by identifying their different secondary structure. Previously, we used nanoIR to analyse patterned lysozyme monomeric and aggregated microdroplets ${ }^{23}$.

In the present work, we employ nanoIR to measure infrared maps and spectra at nanoscale along the whole aggregation pathway of Josephin. We monitor the conversion of monomers into amyloid $\beta$-sheet aggregates and link the intrinsic stiffness of amyloid structures to their $\beta$-sheet content for the first time at the individual species scale. Our results suggest that misfolding in Josephin occurs only after the first aggregation events. nanoIR can shed light on the structural bases of protein fibrillization and misfolding, allowing a deeper comprehension of the protein aggregation field.

\section{Results}

Ultrastructural properties of Josephin aggregates. First, we followed the amyloid aggregation of the Josephin using AFM at 0 , 2 and 7 days of incubation at $37^{\circ} \mathrm{C}$, to set the scenario for further observations. We studied the samples deposited on a $\mathrm{ZnSe}$ surface (Methods). At 0 day, we could observe on this surface monomeric structures with height of $\approx 5 \mathrm{~nm}$ (similar to those evidenced in previous lines of work $^{9}$ ), spheroidal oligomeric species with height up to $\approx 50 \mathrm{~nm}$ and oligomeric clusters with cross-sectional dimensions up to micrometre size (Fig. 1a and Supplementary Fig. 1). After 2 days, we could still observe a similar picture; however, the oligomer species and clusters lost their spheroidal shape (Fig. 1b). At 7 days, there was massive formation of fibrillar aggregates (Fig. 1c-f). A statistical analysis of the height of the smaller oligomeric species, in the AFM maps on the ZnSe prism, showed that their average height increased as a function of the incubation time during the process of fibrillization. The smaller oligomers had an average height of $\sim 5 \mathrm{~nm}$ at 0 day, of $\sim 10 \mathrm{~nm}$ at 2 days and between 10 and $15 \mathrm{~nm}$ at 7 days (Supplementary Fig. 1). The smallest fibrils had an average height of $\sim 10-15 \mathrm{~nm}$ (Fig. 1g). Thus, the height of fibrillar structures corresponded to that of the oligomers. This suggested that aggregation is following the usual process of oligomerization, in agreement with previous studies $^{9}$. Furthermore, the images showed fibrils entangling and forming supramolecular fibrillar aggregates of progressively increasing height and width (Fig. 1d-f), and a quantitative analysis indicated a linear relationship between the height and the convoluted width of these fibrillar structures (Fig. 1g). This suggested that the supramolecular rod-shaped aggregates derive from the association of the isolated population of fibrils with height of 10-15 $\mathrm{nm}$ (ref. 24).

To compare the process of amyloid-structure formation on the $\mathrm{ZnSe}$ prism with the previous experiments in literature on charged substrates ${ }^{9}$, we performed AFM measurements of the same sample solution deposited on a positively functionalized mica substrate (Supplementary Fig. 2). The results were in good agreement with previous experiments and showed that the use of different substrates enables a wider view of the aggregation process in solution (Supplementary Discussion).

To map the evolution of the nanomechanical properties of the Josephin aggregates, we used a fast force-volume system (quantitative imaging (QI)). We produced QI maps of the oligomeric (Fig. 2a,b) and fibrillar (Fig. 2c) structures. Before incubation, the spheroidal oligomers had a uniform Young's modulus of $390 \pm 170 \mathrm{MPa}$ (Fig. 2d). This value is appreciably smaller than what expected for an amyloid structure ${ }^{18,25,26}$. After 2 days of incubation, the sample did not have uniform mechanical properties and two different populations were observed. The first showed stiffness similar to the previous time point $(470 \pm 150 \mathrm{MPa})$, while the latter showed a larger Young's modulus of $0.95 \pm 0.55 \mathrm{GPa}$ (Fig. 2e), a value consistent with formation of a $\beta$-rich structure due to misfolding ${ }^{27,28}$. The final fibrillar structures, after 7 days of incubation, had uniform stiffness of $1.70 \pm 0.65 \mathrm{GPa}$ (Fig. $2 \mathrm{f}$ ), in good agreement with the generally accepted value of stiffness of a mature and complete amyloid cross $\beta$-sheet structure ${ }^{18,25,26}$.

NanoIR of Josephin amyloid species. Although useful, AFM can only provide a morphologic description of the species formed during the fibrillization process but does not tell us whether/at which time point misfolding occurs nor what is the structure of the individual aggregates. Thus, we used nanoIR to characterize further the aggregation pathway. This technique is of particular value when studying non-homogeneous samples, such as amyloid aggregates, offering an important advancement as compared 
with conventional infrared techniques, which, similar to most spectroscopies, averages the structural information over the highly heterogeneous aggregating solution.

We measured the structural properties of Josephin before incubation at $37^{\circ} \mathrm{C}$ (Fig. 3). The images show the morphology (Fig. 3a) and the absorption of infrared light in the amide I $(1,700$ and $1,655 \mathrm{~cm}^{-1}$, Fig. $\left.3 \mathrm{~b}, \mathrm{c}\right)$ and amide III $\left(1,300 \mathrm{~cm}^{-1}\right.$, Fig. $\left.3 \mathrm{~d}\right)$ bands. From the comparison of the absorption maps and considering the components of amide I band related to proteins' secondary structure (Supplementary Discussion), we can infer that the oligomers adsorb mainly infrared radiation in the spectral region of the amide I band, related to random coil and $\alpha$-helical conformations $\left(1,655 \mathrm{~cm}^{-1}\right)$. These chemical absorption maps clearly indicated that the instrument could detect aggregates as small as $50 \mathrm{~nm}$ in average height. This height corresponds, for a hemispherical structure on a surface, to a maximum height of $\sim 100 \mathrm{~nm}$ (Supplementary Discussion and Supplementary Figs 3-4). To confirm quantitatively the trend of absorption shown by infrared, we acquired spectra of individual oligomeric aggregates present on the surface (Fig. 3e). This was achieved by positioning the AFM tip on each structure and collecting several spectra inside their area (minimum 10). The comparison of these spectra showed that they had similar peak amplitudes and positions. The amide I band was approximately within $1,655 \mathrm{~cm}^{-1}\left(\alpha\right.$-helix) and $1,620 \mathrm{~cm}^{-1}$ ( $\beta$-sheet) and centred at $\sim 1,630-1,640 \mathrm{~cm}^{-1}$ (random coil) with a shoulder within $1,720-1,680 \mathrm{~cm}^{-1}$ (antiparallel $\beta$-sheets, $\beta$-turns and side-chain vibrations). The position of the amide II band was approximately within $1,590-1,560 \mathrm{~cm}^{-1}$. However, it was not easily measurable because of low signal-to-noise ratio in this spectral range (Supplementary Discussion and Supplementary Figs 4 and5). The amide III band, centred at $1,300 \mathrm{~cm}^{-1}$, appeared to be coupled with a second peak, which is visible at $1,410-1,400 \mathrm{~cm}^{-1}$. This was already observed in our studies on lysozyme, where we highlighted a change of the relative ratio of the amplitude of this peak and the amide III band during the process of aggregation ${ }^{23}$. This peak can be attributed to a combination of $\mathrm{COO}^{-}, \mathrm{C}-\mathrm{N}, \mathrm{C}-\mathrm{C}$ stretching vibration, $\mathrm{C}-\mathrm{H}$ and $\mathrm{N}-\mathrm{H}$ bending, in plane $\mathrm{O}-\mathrm{H}$ bending. A weaker contribution derives from the vibration of the glutamine $\left(1,410 \mathrm{~cm}^{-1}\right)$, glutamic acid $\left(1,404 \mathrm{~cm}^{-1}\right)$ and aspartic acid $\left(1,402 \mathrm{~cm}^{-1}\right)$ side chain ${ }^{29,30}$.

We considered the averaged deconvolution of the amide I band to estimate the secondary structure content of our uniform spheroidal intermediates (Fig. 3f, Supplementary Table 1 and Supplementary Discussion) $)^{19-21}$. This analysis provided an
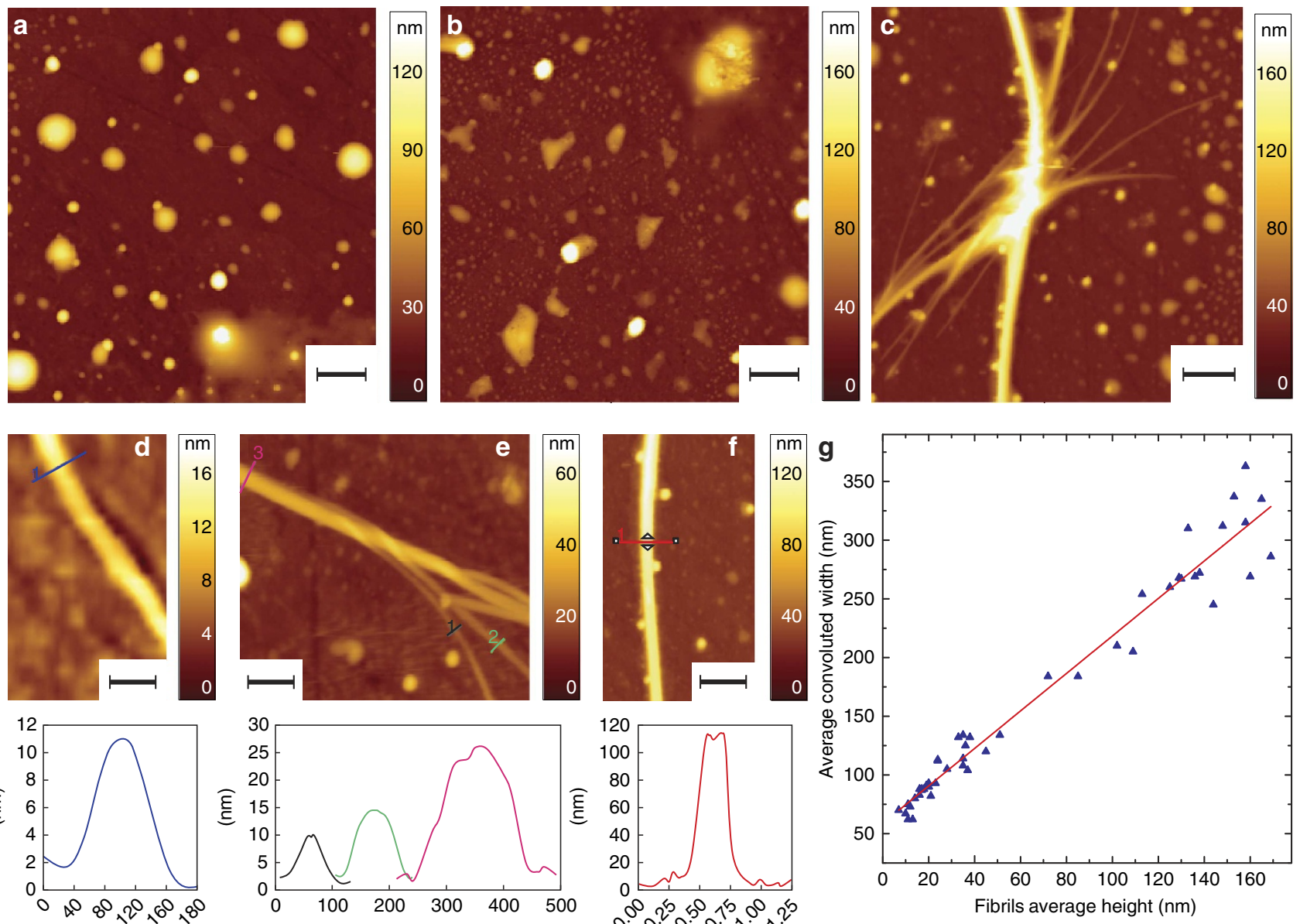

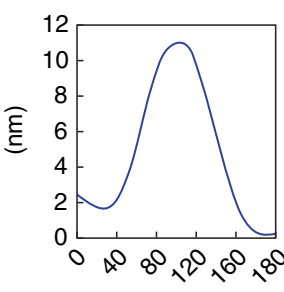

$(\mathrm{nm})$

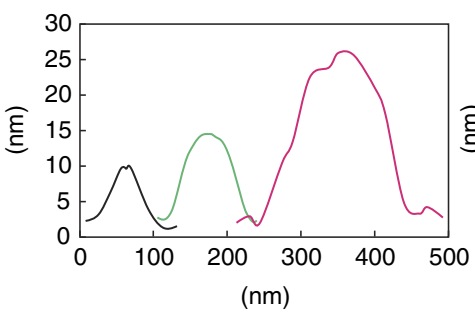

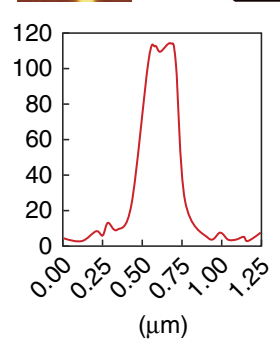

Figure 1 | Josephin's aggregation and fibrillization process. AFM morphology at (a) 0 day, (b) 2 days and (c) 7 days of incubation at $37^{\circ} \mathrm{C}$ (scale bar, $1 \mu \mathrm{m})$. (d) Smaller observable fibrils with height in the order of 10-15 nm (7 days, scale bar, $0.5 \mu \mathrm{m})$. (e) Entangling fibrils with different growing heights (7 days, scale bar, $0.5 \mu \mathrm{m}$ ). (f) Supramolecular aggregate with height of $\sim 115 \mathrm{~nm}$ (7 days, scale bar, $0.5 \mu \mathrm{m}$ ). (g) Analysis of fibrillar morphology: fibrils' average convoluted width as a function of height. The width convolution effect was caused by the finite lateral dimensions of the tip, which contribute to the measured width of the structures. This unavoidable broadening effect is more important when imaging structures with dimensions smaller or comparable with the tip's apical radius $(\approx 10 \mathrm{~nm})$. 
a
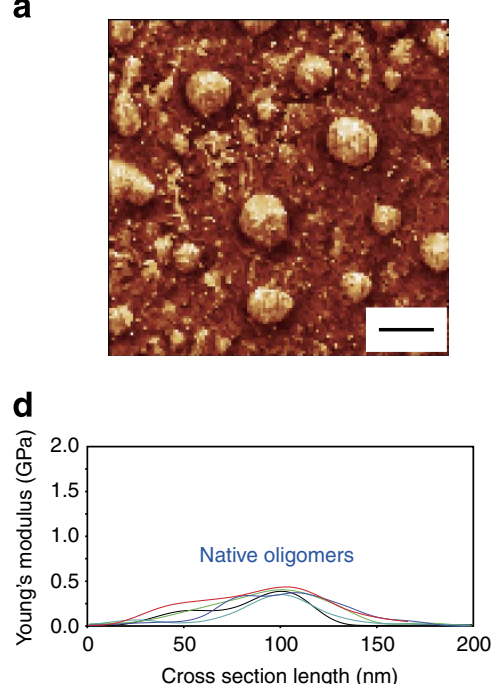

b

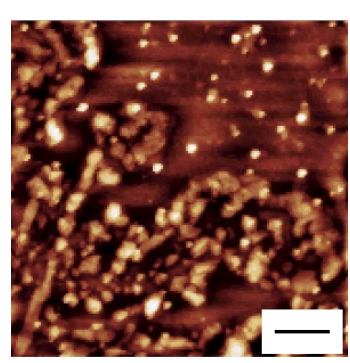

e

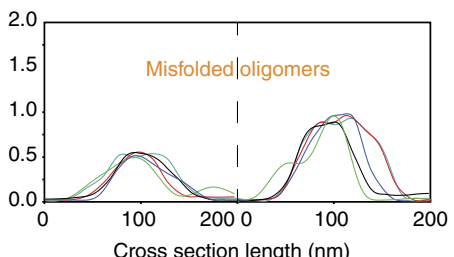

C

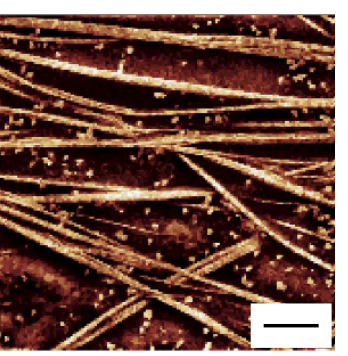

f

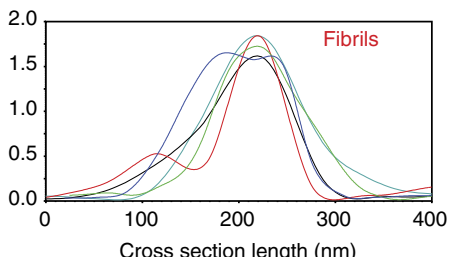

Figure 2 | Young's modulus increases as a function of aggregation. AFM quantitative imaging of: (a) oligomeric proteins at 0 day, (b) oligomers after 2 days and (c) fibrillar structures after 7 days of incubation at $37^{\circ} \mathrm{C}$. Scale bar, $2 \mu \mathrm{m}$. Stiffness cross-sections of (d) oligomers at 0 day, (e) oligomers at 2 days and (f) fibrillar structures.
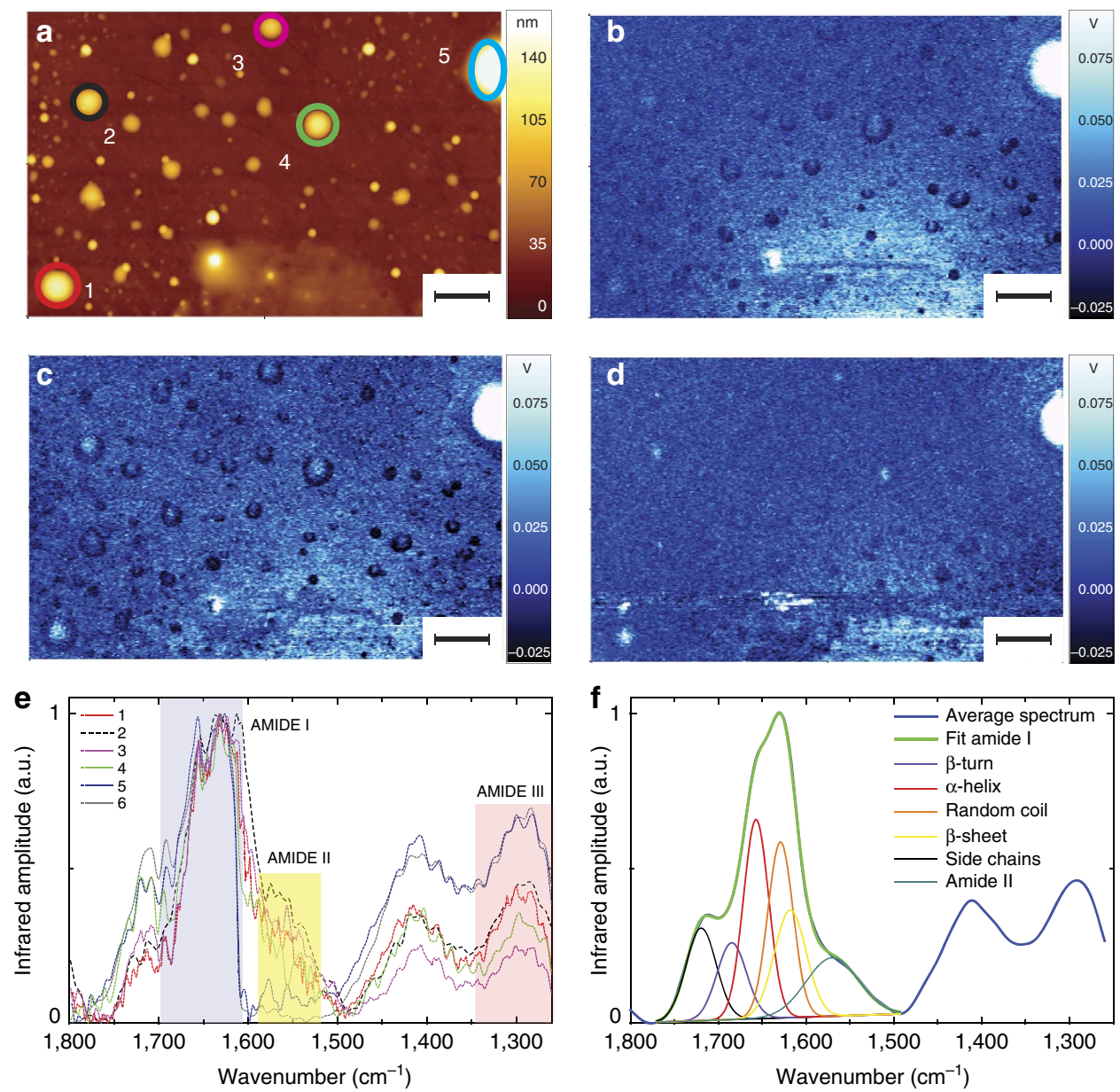

Figure 3 | AFM-infrared chemical maps and spectra of Josephin proteins before incubation at $37^{\circ} \mathbf{C}$. (a) AFM height image. Infrared absorption map at (b) $1,700 \mathrm{~cm}^{-1}$ (amide I), (c) $1,655 \mathrm{~cm}^{-1}$ (amide I), (d) $1,300 \mathrm{~cm}^{-1}$ (amide III). Scale bar, $2 \mu \mathrm{m}$. (e) Infrared spectra. (f) Average oligomeric infrared spectrum and secondary-structure deconvolution of amide I band.

estimate of the secondary structure content of $33 \% \alpha$-helix, $29 \%$ random coil, $23 \% \beta$-turn and $15 \% \beta$-sheet (Supplementary Discussion). These values are in excellent agreement with the monomeric structure of Josephin (35\% $\alpha$-helix, 29\% random coil, $19 \% \beta$-turn and $16 \% \beta$-sheet) and with previously performed bulk Fourier transform infrared spectroscopy 

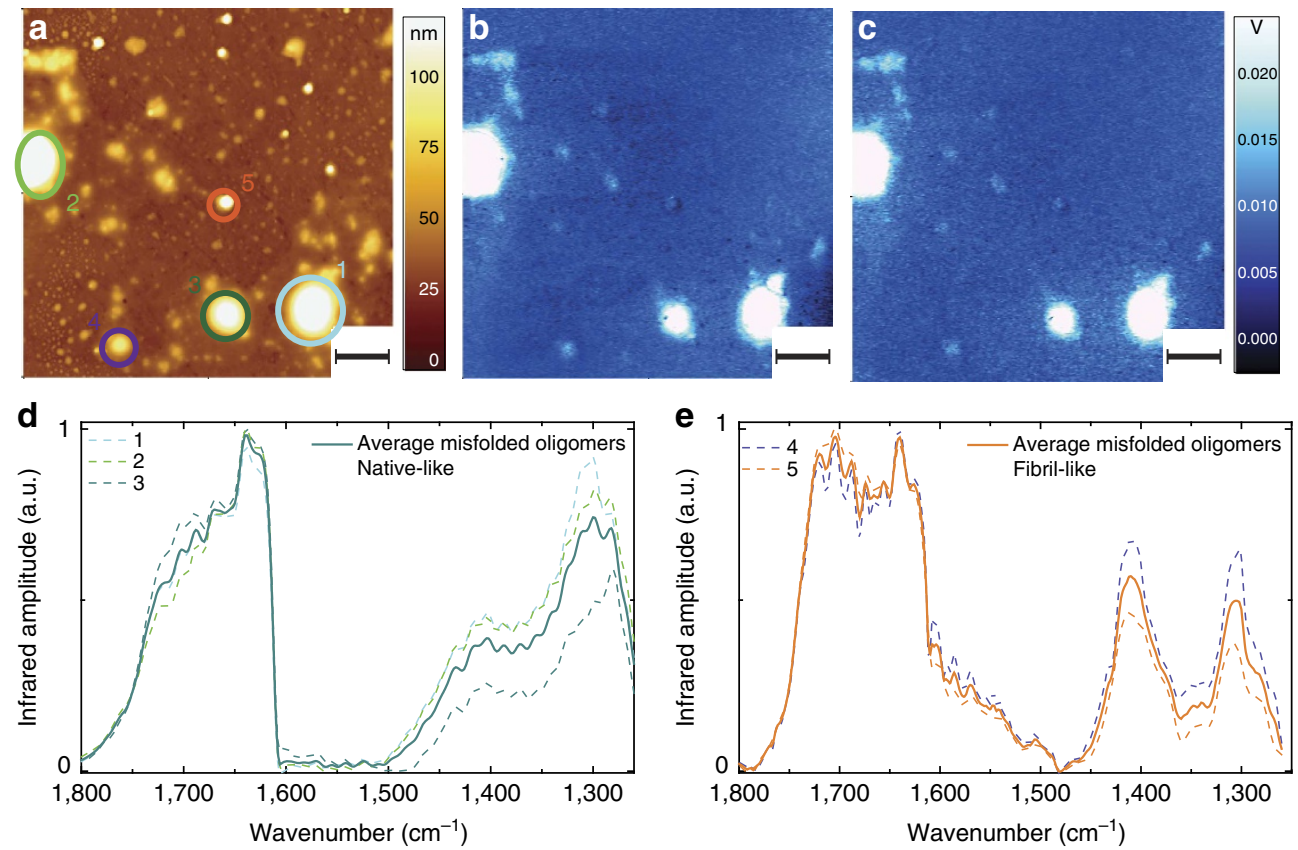

Figure 4 | AFM-infrared chemical maps and spectra after 2-day incubation. (a) AFM height image. Infrared absorption in amide I (b) and at (c) $1,700 \mathrm{~cm}^{-1}$ and (d) $1,655 \mathrm{~cm}^{-1}$. Scale bar, $2 \mu \mathrm{m}$. (d) Spectra of misfolded oligomers, which are native-like with amide I similar to the monomeric structures. (e) Spectra of misfolded oligomers, fibril-like showing a conformational switch towards $\beta$-sheet structure.

measurements ${ }^{10,31,32}$. Thus, before incubation, proteins still retain a native structure.

We repeated the measurements after incubation for 2 days (Fig. 4). As for the 0-day case, we collected the AFM morphology (Fig. 4a) and the infrared absorption maps (Fig. 4b,c). The infrared absorption in the amide I spectral region, between 1,700 and $1,655 \mathrm{~cm}^{-1}$, remained approximately unchanged. Thus, we focused on the spheroidal structures present on the surface and acquired their full infrared spectrum (Fig. 4d,e). We could distinguish two different families of oligomeric structures. The amide I band of the first oligomeric species resonated at frequencies $\left(1,655-1,620 \mathrm{~cm}^{-1}\right)$ similar to the values observed for the spheroidal oligomers observed before incubation. However, the band showed an increased absorption around $1,700 \mathrm{~cm}^{-1}$, which is consistent with an increased content of antiparallel $\beta$-sheet and $\beta$-strand. The shift of the amide I band was more dramatic for the second species, which showed two bands with maxima at 1,700 and $1,640 \mathrm{~cm}^{-1}$, respectively, indicating the disappearance of the $\alpha$-helical component and the increase in the antiparallel $\beta$-sheet content. This was also supported by an inversion of the amplitude ratio between amide III band and the one at $1,410 \mathrm{~cm}^{-1}$.

After 7-day incubation, both oligomers and fibrils were present in the AFM images (Fig. 5a) with typical average heights of $100-150 \mathrm{~nm}$ (Supplementary Fig. 6). Comparison of the absorption maps collected at the amide I band (Fig. 5b,c) and at the band around $1,430 \mathrm{~cm}^{-1}$ (Fig. 5d) showed a clear difference between oligomers and fibrils. The first species had higher absorption in the $\alpha$-helix component of the amide I band $\left(1,655 \mathrm{~cm}^{-1}\right)$. The latter had higher absorption close to the $\beta$-sheet and $\beta$-turn components $\left(1,700-1,680 \mathrm{~cm}^{-1}\right)$ and at $1,430 \mathrm{~cm}^{-1}$. In other words, by performing absorption maps at different wavenumbers, we could clearly distinguish the structure of oligomers from that of fibrils through their different secondary structure content, which was causing differential response to infrared light exposure.

To prove the behaviour shown by the infrared maps, we acquired spectra of individual oligomeric and fibrillar aggregates
(Fig. 5e,f). Overall, the spectra of the fibrils were different from those of the oligomers: they showed a shift of the maximum of the amide I band at $1,700 \mathrm{~cm}^{-1}$ and an inversion of the amplitude ratio within the amide III band and the band around $1,400 \mathrm{~cm}^{-1}$, which had also a net shift towards higher wavenumbers $\left(\sim 1,430 \mathrm{~cm}^{-1}\right)$.

Spectral fingerprint of misfolding and amyloid formation. Finally, we performed principal components analysis (PCA) to prove that the spectral differences measured between the different populations were statistically significant and to extract the spectral fingerprint of amyloid formation. PCA allowed noise reduction, resolution of subtle differences and detection of subgroupings within the spectra.

The most important results of PCA are the scores plots and the loadings plots. The scores plots represent the spectra in the multidimensional space of principal components (PCs), which represent the degree of variability within the ensemble of spectra. The loadings plots show which variables are responsible in the data set for the greatest degree of separation inside this spectra ensemble. The spectral differences are presented in the loadings plots. These determine clustering of spectra in the multidimensional scores plots. For the raw data, the maximum (minimum) of loading is related to the position of a particular vibrational motion, which is typical of the spectra clustered on the positive (negative) values of corresponding PC on the Scores Plot.

The collected spectra were placed in the space of new independent variables, the PCs. Thus, we could distinguish three distinct clusters, corresponding to three groups of aggregates: native oligomers, misfolded oligomers and amyloid fibrils (Fig. 6a). PC-1, which explains $50 \%$ of total variance within the ensemble of spectra (black in Fig. 5b), was positively correlated with the wavenumbers attributed to the amide I in spectral range from 1,710 to $1,680 \mathrm{~cm}^{-1}$, to the $\mathrm{COO}^{-}$vibration around 1,430 $\mathrm{cm}^{-1}$ and to the amide III at $1,285 \mathrm{~cm}^{-1}$ (Fig. 6b). The scores plot showed that these three bands were positively correlated with the PC-1 scores for fibrils and, partially, for misfolded oligomers 

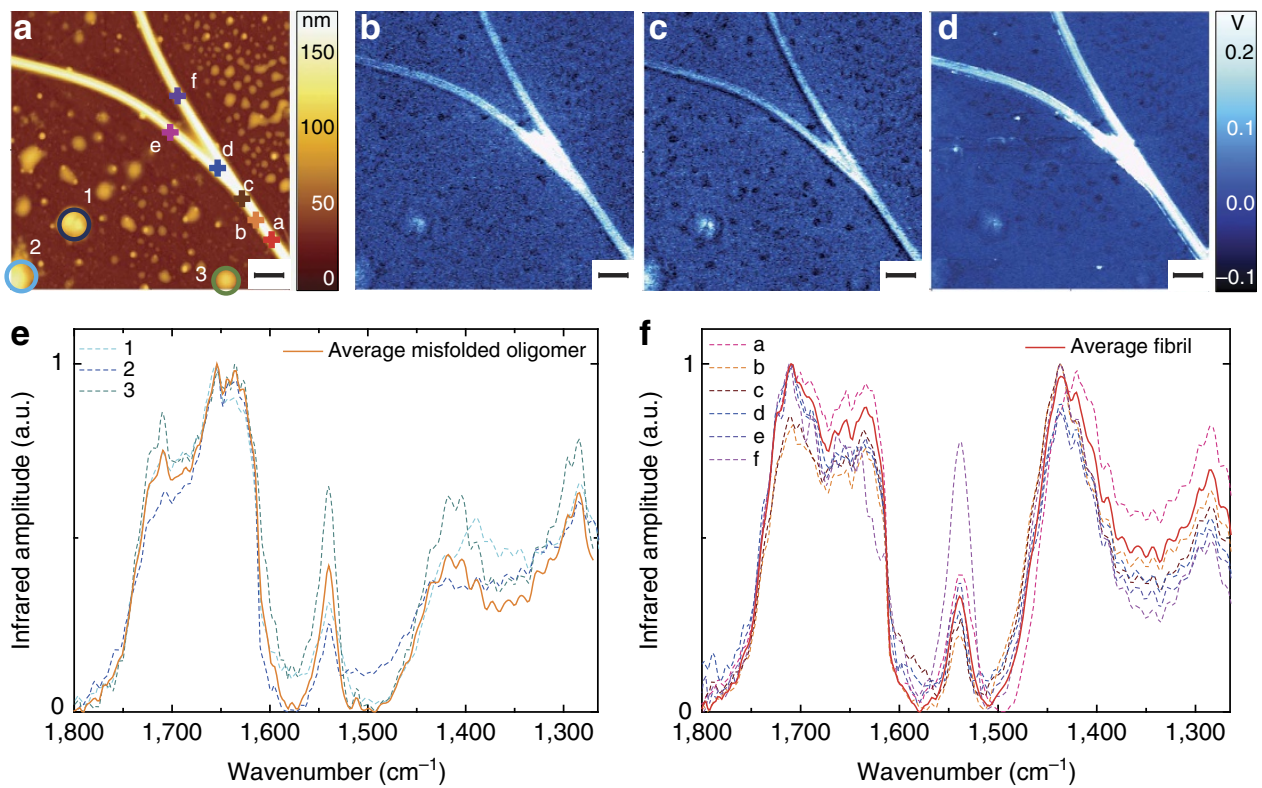

Figure 5 | AFM-infrared chemical maps and spectra of fibrils and oligomers after 7-day incubation. (a) AFM height. Infrared absorption at (b) $1,700 \mathrm{~cm}^{-1}$ (amide I), (c) $1,655 \mathrm{~cm}^{-1}$ (amide I) and (d) $1,430 \mathrm{~cm}^{-1}$. Scale bar, $1 \mu \mathrm{m}$. Spectra of amyloid structures: (e) misfolded oligomer (labelled 1, 2 and 3 in $\mathbf{a}$ ) and (f) fibrils (labelled $a-f$ in $\mathbf{a}$ ).

a

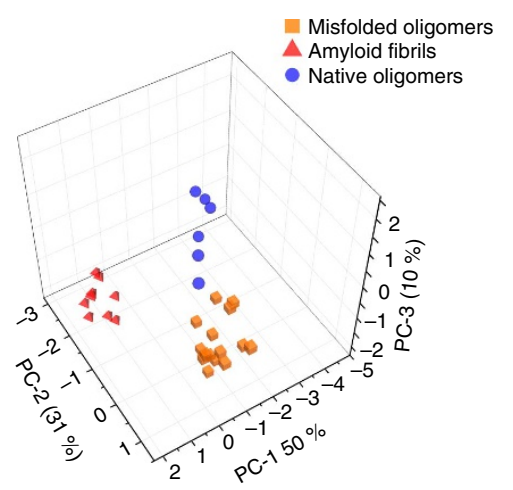

b

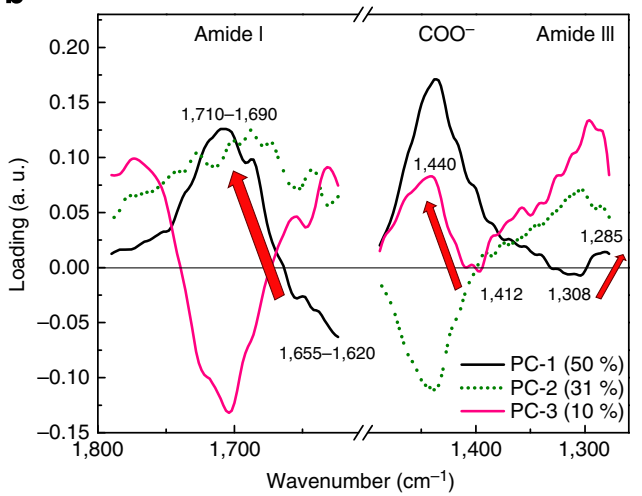

Figure 6 | PCA analysis. The results of PCA analysis in the spectral range 1,800-1,270 $\mathrm{cm}^{-1}$ (excluding 1,620-1,480 $\mathrm{cm}^{-1}$ ) applied to three groups of spectra: native oligomers, misfolded oligomers and fibrils. (a) Three-dimensional scores plot. (b) Loadings plot. The arrows indicate the fingerprint of the conformational changes during amyloid formation.

and negatively correlated with the PC- 1 scores for native oligomers and partially for misfolded oligomers (Fig. 6a). This showed that the amide I band between $1,655-1,620 \mathrm{~cm}^{-1}$ and the amide III band at $1,308 \mathrm{~cm}^{-1}$ are typical of these species. The PC-3, representing $10 \%$ of the total variance, clearly indicated a shift of the $\mathrm{COO}^{-}$band. The $\mathrm{PC}-3$ was positively correlated with the $\mathrm{COO}^{-}$band at $1,430 \mathrm{~cm}^{-1}$ (fibrils and partially misfolded oligomers) and negatively correlated with the band at $1,412 \mathrm{~cm}^{-1}$ (native oligomers and partially misfolded oligomers). We also tested PCA on the second derivatives of the spectra (Supplementary Fig. 7). The conformational change described by the spectra was in good agreement with previous studies in literature using bulk infrared spectroscopy ${ }^{31,32}$. During aggregation, the Fourier transform infrared spectroscopy spectrum changed as a function of time, and the main modifications to the amide I band signal were the decrease in random coil and $\alpha$-helical structure $\left(1,655 \mathrm{~cm}^{-1}\right)$, the increase in antiparallel $\beta$-sheet content $\left(1,695 \mathrm{~cm}^{-1}\right)$ and the decrease in the native $\beta$-sheet content. The results achieved using PCA on second derivatives were well in agreement with the analysis of the raw spectra (Supplementary Discussion and Supplementary Fig. 8).
This analysis confirms statistically that the shift of the amide I band from $1,655-1,620$ to $1,710-1,680 \mathrm{~cm}^{-1}$, the peak at $1,430 \mathrm{~cm}^{-1}$ and the shift of amide III band from 1,308 to $1,295 \mathrm{~cm}^{-1}$ are markers (arrows in the loadings plot Fig. 6b) of the conformational transitions from the random coil/ $\alpha$-helical structure in native oligomers to $\beta$-turn/antiparallel $\beta$-sheet conformations in misfolded oligomers and final amyloid fibrils.

Correlation between nanomechanical and structural properties. The aggregation pathway of Josephin comprises species of large heterogeneity, as we confirmed by using different AFM-based techniques. Morphology measurements showed that fibrillization follows the usual nucleation process of oligomerization. QI forcevolume indicated that the intrinsic stiffness of amyloidogenic species increases as a function of fibrillation maturity (Fig. 7a). A more direct answer on the structural changes that individual amyloid species undergo during aggregation was provided by coupling these results with nanoIR. The initial uniformity of the sample before incubation and the presence of two structurally different oligomeric species at 2 days are well in agreement with the intrinsic stiffness measurements performed using QI. For 

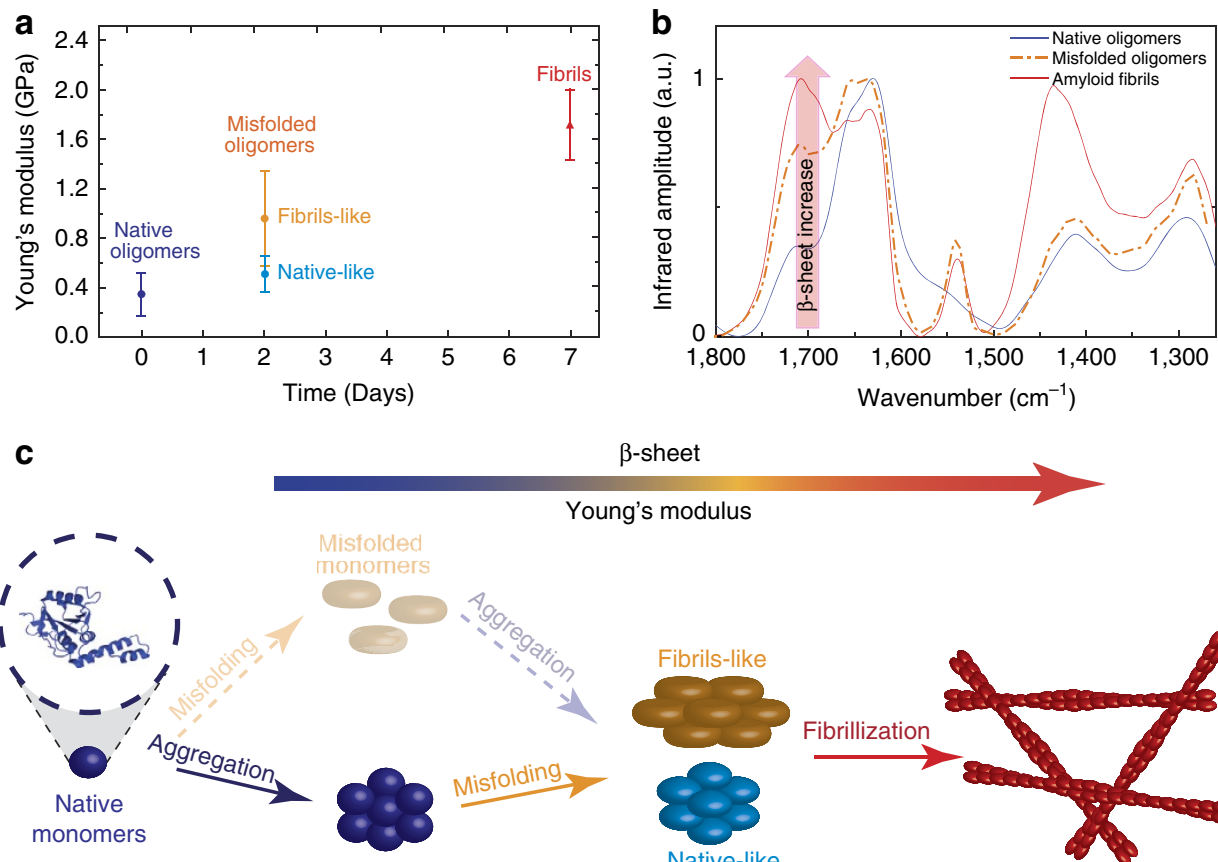

$\beta$-sheet

Young's modulus

Native oligomers Misfolded oligomers

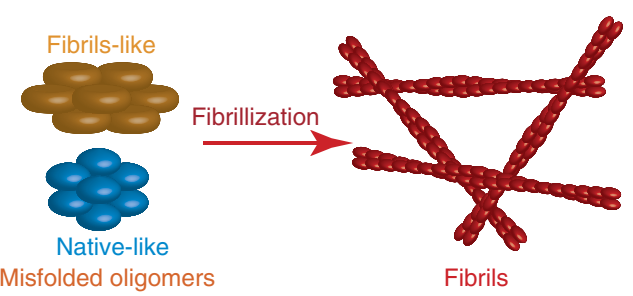

Figure 7 | Model of the link between nanomechanical and structural properties. (a) The increase in the Young's modulus as a function of fibrillization maturity (the error bars are defined as the s.d. of the distribution of the stiffness values of the aggregates). (b) Spectra of native oligomers at 0 day (blue), misfolded oligomers at 2 days (orange and light blue) and amyloid fibrils at 7 days (red). The red arrow indicates the increase in the content of $\beta$-sheet secondary structure. (c) Model of the possible pathways of Josephin aggregation: the transparent model refers to the generally accepted model 'first-misfolding-then-aggregation', while the solid one to the new suggested model of 'first-aggregation-then-misfolding'.

both techniques, we observed two families of misfolded oligomers (Figs 2e and 4). The first has nanomechanical and structural properties similar to those of native oligomers, and we have denominated it native-like. The latter shows an increased stiffness and $\beta$-sheet content, and we called it fibril-like. Finally, the system evolves into a uniform group of fibrillar structures exhibiting the stiffness values and $\beta$-sheet content typical of amyloids (Fig. 7b).

\section{Discussion}

Characterization of all the species formed along the aggregation pathway of a protein is essential in view of the role that protein aggregation has in human health and in material science. In this work, we have investigated, for the first time at the nanoscale, the aggregation pathway of the Josephin domain of ataxin-3, a protein known to be highly fibrillogenic. We could observe directly the conformational transition from native spheroidal oligomers, through misfolded oligomers, to the final amyloid fibrils. We probed the secondary structure of the aggregates down to $50 \mathrm{~nm}$ and demonstrated that, before incubation, the secondary structure of Josephin inside the spheroidal oligomers is clearly native, while misfolding occurs only later, when a conformational transition towards $\beta$-enriched structure occurs. This indicates the intermediate nature of the oligomers, some sharing the structural properties of native monomers (native-like), the others being more similar to fibrils (fibril-like). The fibril-like oligomers form mature fibrils, which have a $\beta$-rich structure and mechanical properties typical of amyloids. This means that we can identify the spectral fingerprint of the $\alpha$-to- $\beta$ transition that native proteins undergo during fibrillization at the nanoscale.

In this way, we correlated the secondary structure of amyloid intermediates and final aggregates to their nanomechanical properties. Indeed, it is central to measure and quantify the ultrastructural properties of amyloid fibrils to appreciate their full potential as biomaterials. It was already suggested that the content of $\beta$-sheets in the aggregates during fibrillization process is an important parameter affecting the nanomechanical properties of these structures. AFM measurements and simulations suggested that the intermolecular interactions (for example, hydrogen bonds) between $\beta$-sheet layers are responsible for the notable mechanical properties of amyloid fibrils ${ }^{33-36}$. Our results directly demonstrated, for the first time at the individual amyloid species scale, that the increase in $\beta$-sheet content is a fundamental parameter determining the growth of amyloids' intrinsic stiffness. Moreover, our evidence strongly suggests that Josephin aggregation precedes misfolding, indicating this protein as an example in which the assumption of 'first-misfolding-and-thenaggregation' may not be true (Fig. $7 \mathrm{c})^{37}$. This possibility is often assumed implicitly or explicitly, mainly because of the observation that misfolding of many globular proteins occurs only on destabilization of the native fold. Such misfolding allows exposure of fibrillogenic sequences otherwise protected in the hydrophobic core. On the other hand, a different pathway to amyloid formation ('first-aggregation-and-then-misfolding') has been more recently identified, in which aggregation of normally globular proteins has been suggested to start from conformational states close to native ones, with no need of transitions across the major energy barrier for unfolding ${ }^{38}$. Our conclusions agree with the evidence that Josephin aggregation is promoted by exposed hydrophobic patches, which have evolved to allow interaction with the non-pathologic cellular partner ubiquitin.

NanoIR proved to be ideal for this study and to characterize individually the species formed along the aggregation pathway, which comprises species of large heterogeneity. We were able to reconstruct the aggregation process and to link nanomechanical and structural properties of individually amyloidogenic species throughout their fibrillization pathway. Conventional methods 
could not have provided this information with the same clarity. This is promising for the development of biomaterials for a broad spectrum of applications in food, nanotechnology and in medicine. Under this perspective, the use of nanoIR can have terrific applications and provide a unique tool to study the aggregation pathway of proteins and help us to design molecules that could interfere with amyloid aggregation.

\section{Methods}

Aggregate preparation. The N-terminal Josephin domain of ataxin-3 (residues 1$182)$ was produced as reported previously ${ }^{39}$. In brief, Josephin was expressed in $E$. coli BL21(DE3) (Life Technologies, USA) as glutathione $S$-transferase (GST) fusion protein with a cleavage site for the tobacco etch virus protease. The GST fusion protein was purified using a glutathione Sepharose affinity matrix (GE Healthcare, UK) and then cleaved from GST using a recombinant His-tagged tobacco etch virus protease. Final purification was achieved using Ni-NTA agarose (Qiagen, Germany). Josephin was incubated at $37^{\circ} \mathrm{C}$ without shaking at a concentration of $14 \mathrm{mM}$, in $20 \mathrm{mM}$ sodium phosphate, $\mathrm{pH} 6.5,10 \mathrm{mM}$ TCEP and $0.025 \% \mathrm{NaN}_{3}$ (all reagents from Sigma-Aldrich, USA) to follow the aggregation pathway. Aliquots of $10 \mu \mathrm{l}$ were taken before and after 2 and 7 days after incubation.

Sample preparation for conventional AFM. We performed conventional AFM measurements in air of the sample deposited on positively functionalized mica. To functionalize the surface, after cleaving, the bare mica substrate was incubated with a $10-\mu l$ drop of $0.05 \%$ (v/v) APTES ((3-Aminopropyl)triethoxysilane, Fluka) in Milli-Q water for $1 \mathrm{~min}$ at room temperature, rinsed with Milli-Q water and then dried by the passage of a gentle flow of gaseous nitrogen. The preparation of the mica AFM samples was realized at room temperature by deposition of a $10-\mu \mathrm{l}$ aliquot of fully concentrated $14-\mu \mathrm{M}$ solution for $10 \mathrm{~min}$. Then the sample was rinsed with ultrapure water and dried by a gentle flow of nitrogen. It is fundamental to underline that these last two passages remove all the material on the surface that is not firmly attached.

QI AFM. We performed all the force-volume stiffness investigations using a JPK Nanowizard III microscope (JPK Instruments AG, Berlin, Germany). The AFM head is working on a commercial inverted optical microscope (Axio Observer.A1, Carl Zeiss, Göttingen, Germany). Analyses were performed using Bruker DNP-10 cantilevers (Bruker probes, Berlin, Germany), choosing the ones with a nominal spring constant of $0.35 \mathrm{~N} \mathrm{~m}^{-1}$. Before each experiment, we calibrated the mechanical properties of the tip using the JPK software. All images were obtained by working in the QI modality, an evolution of the force-volume mode in which the AFM tip is placed in fast oscillation over the sample and the deformation of the cantilever is recorded to reconstruct an image formed by a large number of force distance (FD) curves. Typical images contain up to $256 \times 256$ pixels and, for every pixel, 2,048 points per FD curves were collected. The length of the curves was $300 \mathrm{~nm}$ and the imaging speed ranged from 0.1 to 3 lines per second. The

tip-sample interaction was limited to a maximum cantilever deflection of $3 \mathrm{~nm}$ (that is, corresponding to a maximum applied force of $1 \mathrm{nN}$ ). The data files were recorded on at least five different areas per sample and on minimally 20 different molecules per area. In total, the average values were calculated over $>100$ individual aggregates. Depending on the size and on the resolution of the image, the stiffness of each molecule was measured on a minimum of five points. Processing was carried out in a semi-automated way with the JPK data-processing software assuming that the cantilever behaved accordingly to the Hooke law (that is, the deflection of the cantilever is directly proportional to the vertical component of the force applied on the tip). In this case, the FD curves collected on the sample can be subtracted from the FD curves obtained on the hard substrate, resulting in indentation curves. For these calculations, we assumed a parabolic approximation of the tip shape. The shape of each indentation curve is then used to calculate the mechanical properties of the sample and specifically the stiffness. The average stiffness values, for each condition (monomers, oligomers and fibrils) in Fig. 6a, are expressed in GPa and we defined the error as the s.d. of the measurements on the different structures we analysed.

NanoIR measurements. For all the nanoscale infrared measurements, we used a nanoIR platform (Anasys, CA, USA), which combines high-resolution and low-noise AFM with a tunable infrared laser. This instrument allows nanoscale measurements of infrared absorption as a function of the wavenumber to characterize specimens at spatial resolutions not previously achievable. This approach has been made possible by the photothermal induced resonance effect, also referred to as AFM-infrared ${ }^{40-43}$.

The aliquot samples, diluted at $1 \mu \mathrm{M}$, were deposited at the surface of an attenuated total internal reflection element prism made of $\mathrm{ZnSe}$ monocrystals (Anasys, CA, USA). The ZnSe surface is hydrophobic, as demonstrated by contact angle measurements ${ }^{44}$. This substrate is worldwide accepted and extensively used to investigate amyloid aggregation and conformational change using attenuated total reflection infrared spectroscopy ${ }^{21,22,45}$. Then, we dried the samples by evaporation, so that all the proteins in solution were deposited on the surface.
After the preparation, we scanned the samples using the nanoIR microscopy system, with a rate line within $0.08-0.04 \mathrm{~Hz}$ and in contact mode. We used a silicon (AppNano, CA, USA) cantilever with a nominal radius of $10 \mathrm{~nm}$ and an elastic constant of $\sim 0.5 \mathrm{~N} \mathrm{~m}^{-1}$. All images have a resolution of at least $1,024 \times 512$ pixels per line. The AFM images were treated using the SPIP software. The height and infrared amplitude images were first-order flattened. The spectra were collected with a sampling of $1 \mathrm{~cm}^{-1}$ and $256 \mathrm{co}$-averages, within the range $1,200-1,800 \mathrm{~cm}^{-1}$ and with a spectral resolution of $8 \mathrm{~cm}^{-1}$. Spectra were averaged from at least 10 measurements. They were normalized using the microscope's built-in Anasys software (Analysis Studio) and OriginPRO. Successively, they were smoothed with a Savitzky-Golay filter (second order, nine points).

All measurements were performed at room temperature. The ultimate resolution of the instrument allows detecting objects with thickness of $50-100 \mathrm{~nm}$ and with a lateral resolution as small as the radius of the AFM tip. This minimal measurable thickness is defined by the minimal detectable photothermal expansion of the sample. On the other hand, the lateral resolution of an isolated object is only limited by the sharpness of the AFM lever. In the case of non-isolated objects, thermal diffusion can limit lateral spatial resolution.

Principal component analysis. We performed the PCA by means of the nonlinear iterative partial least squares algorithm with cross-validation and mean-centred data, using the Unscrambler 9.2 software (CAMO, Norway). Analysis was performed on underivatized spectra and second derivatives. Second derivatives were calculated with the Unscrambler 9.2 software using the Savitzky-Golay algorithm for smoothing (second order, 9 smoothing points). The amide II region $\left(1,620-1,480 \mathrm{~cm}^{-1}\right)$ was excluded from the analysis because of low signal-to-noise ratio in this spectral range, as mentioned earlier.

\section{References}

1. Hardy, J. \& Selkoe, D. J. The amyloid hypothesis of Alzheimer's disease: progress and problems on the road to therapeutics. Science 297, 353-356 (2002).

2. Chiti, F. \& Dobson, C. M. Protein misfolding, functional amyloid, and human disease. Annu. Rev. Biochem. 75, 333-366 (2006).

3. Chapman, M. R. et al. Role of Escherichia coli curli operons in directing amyloid fiber formation. Science 295, 851-855 (2002).

4. Fowler, D. M., Koulov, A. V., Balch, W. E. \& Kelly, J. W. Functional amyloid - from bacteria to humans. Trends Biochem. Sci. 32, 217-224 (2007).

5. Shewmaker, F., McGlinchey, R. P. \& Wickner, R. B. Structural Insights into Functional and Pathological Amyloid. J. Biol. Chem. 286, 16533-16540 (2011).

6. Sunde, M. et al. Common core structure of amyloid fibrils by synchrotron X-ray diffraction. J. Mol. Biol. 273, 729-739 (1997).

7. Pedersen, J. S., Andersen, C. B. \& Otzen, D. E. Amyloid structure-one but not the same: the many levels of fibrillar polymorphism. FEBS J. 277, 4591-4601 (2010).

8. Greenwald, J., Rol, Riek \& Riek, R. Biology of amyloid: structure, function, and regulation. Structure 18, 1244-1260 (2010).

9. Masino, L. et al. The Josephin domain determines the morphological and mechanical properties of ataxin-3 fibrils. Biophys. J. 100, 2033-2042 (2011).

10. Masino, L. et al. Characterization of the structure and the amyloidogenic properties of the Josephin domain of the polyglutamine-containing protein ataxin-3. J. Mol. Biol. 344, 1021-1035 (2004).

11. gales, l. et al. Towards a structural understanding of the fibrillization pathway in machado-Joseph's disease: trapping early oligomers of non-expanded ataxin-3. J. Mol. Biol. 353, 642-654 (2005).

12. Ellisdon, A. M., Pearce, M. C. \& Bottomley, S. P. Mechanisms of ataxin-3 misfolding and fibril formation: kinetic analysis of a disease-associated polyglutamine protein. J. Mol. Biol. 368, 595-605 (2007).

13. Ellisdon, A. M., Thomas, B. \& Bottomley, S. P. The two-stage pathway of ataxin-3 fibrillogenesis involves a polyglutamine-independent step. J. Biol. Chem. 281, 16888-16896 (2006).

14. Dazzi, A. et al. AFM-IR: combining atomic force microscopy and infrared spectroscopy for nanoscale chemical characterization. Appl. Spectrosc. 66, 1365-1384 (2012).

15. Lahiri, B., Holland, G. \& Centrone, A. chemical imaging beyond the diffraction limit: experimental validation of the PTIR technique. Small 9, 439-445 (2013).

16. Adamcik, J. et al. Understanding amyloid aggregation by statistical analysis of atomic force microscopy images. Nat. Nanotechnol. 5, 423-428 (2010).

17. Adamcik, J. \& Mezzenga, R. Study of amyloid fibrils via atomic force microscopy. Curr. Opin. Colloid Interface Sci. 17, 369-376 (2012).

18. Adamcik, J. et al. Measurement of intrinsic properties of amyloid fibrils by the peak force QNM method. Nanoscale 4, 4426 (2012).

19. Jackson, M. \& Mantsch, H. The use and misuse of FTIR spectroscopy in the determination of protein structure. Crit. Rev. Biochem. 30, 95-120 (1995).

20. Zandomeneghi, G., Krebs, M. R., McCammon, M. G. \& Fändrich, M. FTIR reveals structural differences between native $\beta$-sheet proteins and amyloid fibrils. Protein Sci. 13, 3314-3321 (2004). 
21. Calero, M. \& Gasset, M. Featuring amyloids with Fourier transform infrared and circular dichroism spectroscopies. Methods Mol. Bio. 849, 53-68 (2012).

22. Sarroukh, R., Goormaghtigh, E., Ruysschaert, J.-M. \& Raussens, V. ATR-FTIR: a "rejuvenated" tool to investigate amyloid proteins. Biochim. Biophys. Acta Biomembranes 1828, 2328-2338 (2013).

23. Muller, T. et al. Nanoscale spatially resolved infrared spectra from single microdroplets. Lab. Chip 14, 1315-1319 (2014).

24. Ridgley, D. M. \& Barone, J. R. Evolution of the amyloid fiber over multiple length scales. ACS Nano. 7, 1006-1015 (2013).

25. Knowles, T. P. \& Buehler, M. J. Nanomechanics of functional and pathological amyloid materials. Nat. Nanotechnol. 6, 469-479 (2011).

26. Smith, J. F., Knowles, T. P., Dobson, C. M., MacPhee, C. E. \& Welland, M. E. Characterization of the nanoscale properties of individual amyloid fibrils. Proc. Natl Acad. Sci. USA 103, 15806-15811 (2006).

27. Uversky, V. N., Li, J. \& Fink, A. L. Evidence for a partially folded intermediate in alpha-synuclein fibril formation. J. Biol. Chem. 276, 10737-10744 (2001).

28. Hong, D., Fink, A. L. \& Uversky, V. N. Structural characteristics of $\alpha$-synuclein oligomers stabilized by the flavonoid baicalein. J. Mol. Biol. 383, 214-223 (2008).

29. Barth, A. Infrared spectroscopy of proteins. Biochim. Biophys. Acta 1767, 1073-1101 (2007).

30. Nicastro, G., Habeck, M., Masino, L., Svergun, D. I. \& Pastore, A. Structure validation of the Josephin domain of ataxin-3: conclusive evidence for an open conformation. J. Biomol. NMR 36, 267-277 (2006).

31. Natalello, A. et al. A major role for side-chain polyglutamine hydrogen bonding in irreversible ataxin-3 aggregation. PLoS ONE 6, e18789 (2011).

32. Santambrogio, C. et al. The role of the central flexible region on the aggregation and conformational properties of human ataxin-3. FEBS J. 279, 451-463 (2012).

33. Paparcone, R. \& Buehler, M. J. Microscale structural model of Alzheimer A beta(1-40) amyloid fibril. Appl. Phys. Lett. 94, 243904 (2009).

34. Xu, Z. P., Paparcone, R. \& Buehler, M. J. Alzheimer's A beta(1-40) amyloid fibrils feature size-dependent mechanical properties. Biophys. J. 98, 2053-2062 (2010).

35. Paparcone, R., Keten, S. \& Buehler, M. J. Atomistic simulation of nanomechanical properties of Alzheimer's A beta(1-40) amyloid fibrils under compressive and tensile loading. J. Biomech. 43, 1196-1201 (2010).

36. Paparcone, R., Cranford, S. W. \& Buehler, M. J. Self-folding and aggregation of amyloid nanofibrils. Nanoscale 3, 1748-1755 (2011).

37. Masino, L., Nicastro, G., Calder, L., Vendruscolo, M. \& Pastore, A. Functional interactions as a survival strategy against abnormal aggregation. FASEB J. 25, 45-54 (2011).

38. Bemporad, F. \& Chiti, F. 'Native-like aggregation' of the acylphosphatase from Sulfolobus solfataricus and its biological implications. FEBS Lett. 583, 2630-2638 (2009)

39. Nicastro, G. et al. The solution structure of the Josephin domain of ataxin-3: structural determinants for molecular recognition. Proc. Natl Acad. Sci. USA 102, 10493-10498 (2005)
40. Katzenmeyer, A. M., Aksyuk, V. \& Centrone, A. Nanoscale infrared spectroscopy: improving the spectral range of the photothermal induced resonance technique. Anal. Chem. 85, 1972-1979 (2013).

41. Nasse, M. J. et al. High-resolution Fourier-transform infrared chemical imaging with multiple synchrotron beams. Nat. Methods 8, 413-416 (2011).

42. Dazzi, A., Glotin, F. \& Carminati, R. Theory of infrared nanospectroscopy by photothermal induced resonance. J. Appl. Phys. 107, 124519 (2010).

43. Dazzi, A. et al. Chemical mapping of the distribution of viruses into infected bacteria with a photothermal method. Ultramicroscopy 108, 635-641 (2008).

44. Jeon, J. S., Raghavan, S. \& Sperline, R. P. Quantitative analysis of albumin adsorption onto uncoated and poly(ether)urethane-coated ZnSe surfaces using the attenuated total reflection FTIR technique. Colloids Surf. A 92, 255-265 (1994).

45. Shivu, B. et al. Distinct $\beta$-Sheet structure in protein aggregates determined by ATR-FTIR spectroscopy. Biochemistry 52, 5176-5183 (2013).

\section{Acknowledgements}

We thank the Swiss National Foundation for Science (grant number 152958) and the Medical Research Council (U117584256) for the support.

\section{Author contributions}

F.S.R. and A.P. conceived and planned the experiments. S.F. prepared biological samples F.S.R. prepared samples and performed infrared nanospectroscopy measurements and analysis. G.L. conducted nanomechanical measurements. E.L. performed principal component analysis. F.S.R., G.L., E.L., A.P. and G.D. wrote the manuscript. All the authors commented on the manuscript.

\section{Additional information}

Supplementary Information accompanies this paper at http://www.nature.com/ naturecommunications

Competing financial interests: The authors declare no competing financial interests.

Reprints and permission information is available online at http://npg.nature.com/ reprintsandpermissions/

How to cite this article: Ruggeri, F. S. et al. Infrared nanospectroscopy characterization of oligomeric and fibrillar aggregates during amyloid formation. Nat. Commun. 6:7831 doi: $10.1038 /$ ncomms8831 (2015)

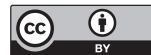

This work is licensed under a Creative Commons Attribution 4.0 International License. The images or other third party material in this article are included in the article's Creative Commons license, unless indicated otherwise in the credit line; if the material is not included under the Creative Commons license, users will need to obtain permission from the license holder to reproduce the material. To view a copy of this license, visit http://creativecommons.org/licenses/by/4.0/ 